\title{
Correction to: A Regular Integral Equation Formalism for Solving the Standard Boussinesq's Equations for Variable Water Depth
}

\section{T. S. Jang ${ }^{1}$}

Published online: 18 November 2019

(c) The Author(s) 2019

\section{Correction to: J Sci Comput (2018) 75:1721-1756 https://doi.org/10.1007/s10915-017-0605-6}

The author would like to correct an error in calculation of the Eq. (A.2.8) in Appendix A.2 of the original article.

The correct equation is as follows:

$$
\begin{aligned}
\mathrm{U}_{x t}^{(4)}(b)(x, t)= & \frac{\partial^{2}}{\partial x \partial t} \mathrm{U}^{(4)}(b)(x, t)=\frac{\partial}{\partial t}\left\{\mathrm{U}_{x}^{(4)}(b)\right\} \\
= & \frac{1}{\pi} \frac{\partial}{\partial t} \int_{0}^{\infty} \int_{0}^{t} \int_{-\infty}^{\infty} \frac{k}{1+h_{0}^{2} k^{2} / 3} \cdot \cos \left[\omega_{\mathrm{B}}(t-\tau)\right] \cdot \sin [k(\xi-x)] \cdot b(\xi, \tau) d \xi d \tau d k \\
= & \frac{1}{\pi} \int_{0}^{\infty} \int_{0}^{t} \int_{-\infty}^{\infty} \frac{k}{1+h_{0}^{2} k^{2} / 3} \cdot \frac{\partial}{\partial t} \cos \left[\omega_{\mathrm{B}}(t-\tau)\right] \cdot \sin [k(\xi-x)] \cdot b(\xi, \tau) d \xi d \tau d k \\
& \left.+\frac{1}{\pi} \int_{0}^{\infty}\left[\int_{-\infty}^{\infty} \frac{k}{1+h_{0}^{2} k^{2} / 3} \cdot \cos \left[\omega_{\mathrm{B}}(t-t)\right] \cdot \sin [k(\xi-x)] \cdot b(\xi, \tau=t) d \xi\right\} \frac{d t}{d t}\right] d k \\
= & -\frac{1}{\pi} \int_{0}^{t} \int_{0}^{\infty} \int_{-\infty}^{\infty} \frac{k \omega_{\mathrm{B}}}{1+h_{0}^{2} k^{2} / 3} \cdot \sin \left[\omega_{\mathrm{B}}(t-\tau)\right] \cdot \sin [k(\xi-x)] \cdot b(\xi, \tau) d \xi d k d \tau \\
& +\frac{1}{\pi} \int_{0}^{\infty} \int_{-\infty}^{\infty} \frac{k}{1+h_{0}^{2} k^{2} / 3} \cdot \sin [k(\xi-x)] \cdot b(\xi, t) d \xi d k
\end{aligned}
$$

Open Access This article is distributed under the terms of the Creative Commons Attribution 4.0 International License (http://creativecommons.org/licenses/by/4.0/), which permits unrestricted use, distribution, and reproduction in any medium, provided you give appropriate credit to the original author(s) and the source, provide a link to the Creative Commons license, and indicate if changes were made.

Publisher's Note Springer Nature remains neutral with regard to jurisdictional claims in published maps and institutional affiliations.

The original article can be found online at https://doi.org/10.1007/s10915-017-0605-6.

\section{T. S. Jang}

taek@pusan.ac.kr

1 Department of Naval Architecture and Ocean Engineering, Pusan National University, Busan 46241, Republic of Korea 